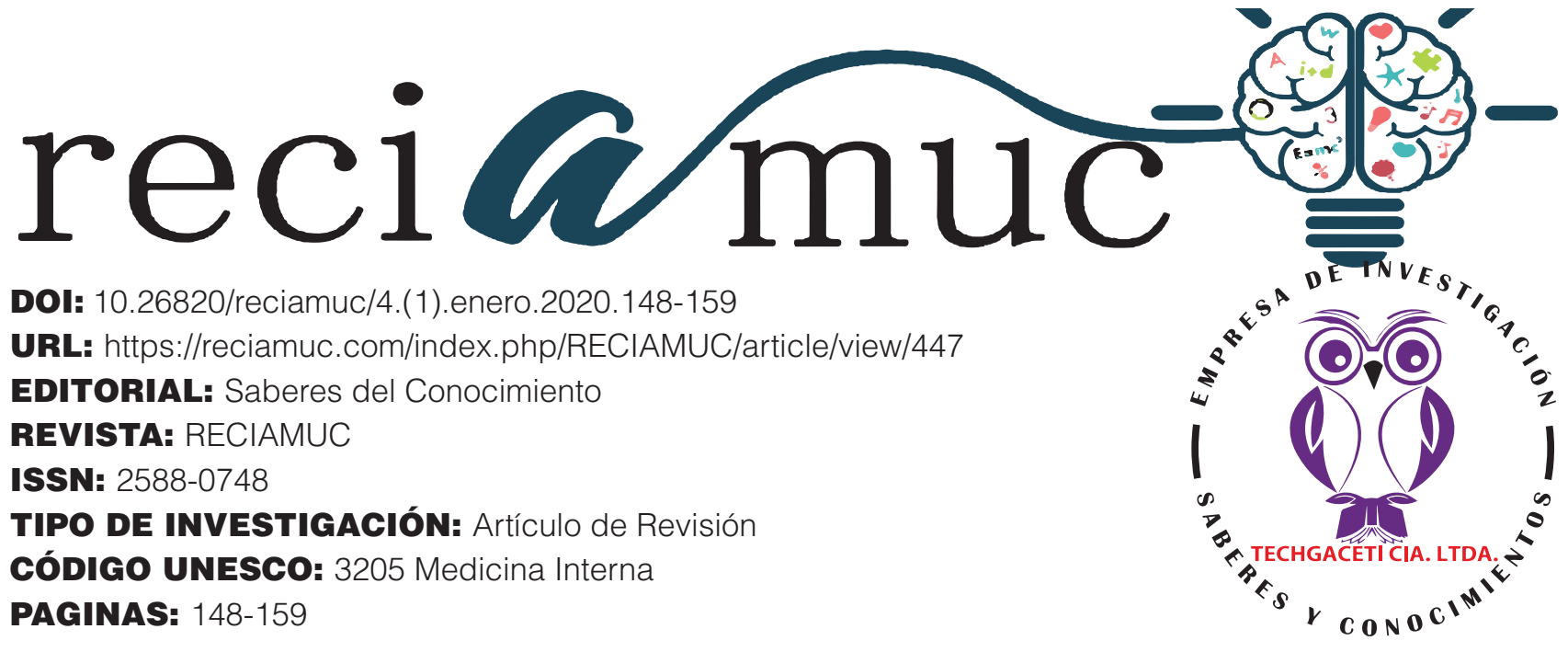

\title{
Encefalopatía urémica. Causas y tratamiento
}

\author{
Uremic encephalopathy. Causes and treatment
}

Encefalopatia urêmica. Causas e tratamento

Juan Sebastian de Mora Flores'; Yomara Catherine Benítez Ormaza²; Elizabeth del Rocío Gavilánez Toalombo 3 ; Evelyn Anette Alvarez Perez ${ }^{4}$

\section{RECIBIDO: 18/11/2019 ACEPTADO: 20/12/2019 PUBLICADO: 31/01/2020}

1. Médico; Investigador Independiente; Guayaquil, Ecuador; sebastian_demora@hotmail.com; (D) https://orcid. org/0000-0001-5493-3955

2. Médico; Investigador Independiente; Guayaquil, Ecuador; yomarabenitezo@gmail.com; (D) https://orcid. org/0000-0002-8232-1646

3. Médico General; Investigador Independiente; Guayaquil, Ecuador; eliznega_12@hotmail.es; (D) https://orcid. org/0000-0003-0437-327X

4. Médico Cirujana; Investigador Independiente; Guayaquil, Ecuador; evelynalvarez0193@gmail.com ; (DD https:// orcid.org/0000-0003-4642-2746

CORRESPONDENCIA

Juan Sebastian de Mora Flores

sebastian_demora@hotmail.com

Guayaquil, Ecuador

@ RECIAMUC; Editorial Saberes del Conocimiento, 2019 


\section{RESUMEN}

La encefalopatía urémica como su nombre lo índica son aquellas alteraciones en el estado de conciencia, se refiere a esta como síndrome del encéfalo, originado como consecuencia de otra patología principal, tal como se manifiesta las demás encefalopatías conocidas. En este caso, producto de la insuficiencia renal se origina esta encefalopatía, que hoy obliga a la investigación científica, de sus posibles causas, sus síntomas y manifestaciones, así como los tratamientos para minimizar los efectos de esta enfermedad que es previsible y sobre la cual puede revertirse los efectos que incluso pueden llevar a los estado de gravedad como el coma y hasta a la muerte.

En efecto, esta enfermedad, requiere de estudios minuciosos y de diagnósticos tempranos para evitar los síntomas severos que serán parte del análisis a continuación. Esta investigación se propone ser una revisión documental, que motive las investigaciones científicas de este síndrome y que genere aporte al estudio de esta patología.

Palabras clave: Encéfalo, Encefalopatía Urémica, Insuficiencia Renal, Síndrome Urémico, Diálisis, Trasplante Renal, Complicaciones.

\section{ABSTRACT}

Uremic encephalopathy, as its name indicates, are those alterations in the state of consciousness, it refers to this as a brain syndrome, originated as a consequence of another main pathology, as the other known encephalopathies manifest. In this case, as a result of renal failure, this encephalopathy originates, which today forces scientific research, of its possible causes, its symptoms and manifestations, as well as treatments to minimize the effects of this disease that is predictable and on which the effects that can even lead to the state of gravity such as coma and even death can be reversed.

Indeed, this disease requires thorough studies and early diagnoses to avoid severe symptoms that will be part of the analysis below. This research aims to be a documentary review, which motivates the scientific investigations of this syndrome and that generates contribution to the study of this pathology.

Keywords: Brain, uremic encephalopathy, renal failure, uremic syndrome, dialysis, kidney transplantation, complications.

\section{RESUMO}

A encefalopatia urêmica, como o próprio nome indica, são aquelas alterações no estado de consciência, refere-se a isso como uma síndrome cerebral, originada como consequência de outra patologia principal, à medida que as outras encefalopatias conhecidas se manifestam. Nesse caso, como resultado da insuficiência renal, é originada essa encefalopatia, que hoje força a pesquisa científica de suas possíveis causas, sintomas e manifestações, além de tratamentos para minimizar os efeitos previsíveis e sobre os quais essa doença é previsível. os efeitos que podem levar ao estado de gravidade, como coma e até a morte, podem ser revertidos.

De fato, esta doença requer estudos completos e diagnóstico precoce para evitar sintomas graves que farão parte da análise abaixo. Esta pesquisa pretende ser uma revisão documental, que motive as investigações científicas dessa síndrome e que gere contribuição para o estudo dessa patologia.

Palavras-chave: Cérebro, encefalopatia urêmica, insuficiência renal, síndrome urêmica, diálise, transplante renal, complicações. 


\section{Introducción}

El diagnóstico de la encefalopatía urémica esta basado principalmente en las alteraciones neurológicas presentes en pacientes con insuficiencia renal, cuando son en ausentes causas evidentes de enfermedad cerebral; sin embargo actualmente las causas de la encefalopatía urémica son desconocidas. El tratamiento temprano de muchos tipos de encefalopatía puede eliminar, reducir o detener los síntomas de la enfermedad. En muchas ocasiones, se puede prevenir evitando las causas primarias.

Los mecanismos sugeridos incluyen depresión del consumode oxígeno cerebral, hipoxia cerebral, incremento en los niveles cerebrales de calcio, toxinas urémicas (metilguanidina, ácido guanidino succínico, ácido fenólico) y elevación en los niveles sanguíneos de parathormona. Todos estos de acuerdo al tipo de encefalopatía.

En este sentido, el siguiente estudio, analiza la generalidad de las encefalopatías, el síndrome urémico y sus especificidades y en consecuencias determinar los preceptos fundamentales que originan a la Encefalopatía urémica, en aras de orientar sobre su diagnostico oportuno, sus tratamientos y formas preventivas, debido a que la misma puede agravarse y causar la muerte. En efecto, el análisis se detendrá en las causas que la originan, y en los tratamientos mas comúnmente usados, previo a su evolución a estados de mayor gravedad.

\section{Método}

La metodología que se presenta en este estudio es de tipo documental con revisión y análisis de bibliografía, la recopilación de la información se ha elaborado a través de una investigación documental. Más de 50 artículos revisados en diferentes medios bibliográficos, medios Electrónicos, diccionarios médicos, libros, artículos de revistas y otros, en función de generar aportes teóricos en cuando a las encefalopatías.
Fueron considerados los aportes más importantes de ciertos autores con criterio de selección de los planteamientos teóricos comunes entre los a especialistas analizados, y se plantea la investigación para futuros casos que den soporte a nuevos estudios.

\section{Encefalopatía causas y tratamiento}

Cuando se habla de Encefalopatía este término refiere al desorden o enfermedad del encéfalo. Encefalopatía como síndrome refiere a la disfunción cerebral, el cual puede ser causado por múltiples etiologías.LA encefalopatía, está precedido por varios términos que describen su origen etimológico, (la razón, causa, o condiciones especiales del paciente); tal es el caso de la encefalopatía anóxica significa daño cerebral por falta de oxígeno, así en total, existen más de 150 tipos de encefalopatía que describen la literatura médica de este síndrome.

La Encefalopatía "Es un síndrome orgánico cerebral agudo o subagudo que ocurre en pacientes con insuficiencia renal aguda o crónica, cuando la fi Itración glomerular cae por debajo del 10 \% de lo normal" (Rondón Nucete, 1998)

Infecciones, problemas metabólicos, toxinas, medicamentos, cambios fisiológicos y traumatismos, son algunas de las causas que se atribuyen a la encefalopatía. Es además considerada como una complicación de un problema principal en particular, tal es el caso de cirrosis hepática, insuficiencia renal, o anoxia; de allí que el tratamiento preventivo o a tiempo de muchos de los tipos de encefalopatía puede reducir y hasta detener los síntomas de la enfermedad.

Las Encefalopatías en general, son complicaciones halladas en pacientes con alteración en sus estado de conciencia, hospitalizado o manifestadas en Unidad de Cuidados Intensivos, tal como lo plantea (Navas \& González, 2004): "Existe mayor morbibilidad, estadía hospitalización y mortalidad en los pacientes críticamente enfermos que desarrollan complicaciones neuro- 
lógicas". De tal manera que su diagnostico oportuno para proceder con tratamiento eficaz puede prever estados de mayor gravedad en los pacientes.

Los síntomas pueden manifestarse como pérdidas de memorias, hasta demencia, convulsiones, estado de coma o la muerte, en efecto se plantea síntomas de amplio espectropero que en definitiva son reducto de un estado mental alterado algunas veces con manifestaciones físicas.

En el caso de encefalopatia Uremica los síntomas son reconocidos comunmente y plasmado por (Cuddon, 1996).como "alteraciones cognitivas, neuromusculares, somato sensoriales y autonómicas. En general, la gravedad y progresión de los signos neurológicos es proporcional al deterioro de la función renal y a su velocidad de instauración."Los síntomas urémicos suelen ser más graves y avanzan más rápidamente en los pacientes con insuficiencia renal aguda que en aquellos con insuficiencia renal crónica

Causas de las Encefalopatía: De manera general todas las encefalopatías pueden distinguir las siguientes causas:

- Anoxia (falta de oxígeno en el cerebro, incluyendo causas traumáticas).

- Infecciones (bacterias, virus, parásitos).

- Alcoholismo (toxicidad del alcohol).

- Condiciones hepáticas (por ejemplo, insuficiencia hepática o cáncer de hígado).

- Trastornos renales (insuficiencia renal).

- Enfermedades metabólicas (hiper o hipocalcemia, hipo o hipernatremia o hipo o hiperglucemia).

- Tumores cerebrales.

- Algunos tipos de productos químicos tóxicos (mercurio, plomo, o amoníaco).

- Alteraciones en la presión dentro del ce- rebro (a menudo asociadas con sangrado, tumores o abscesos).

- Ingesta inadecuada de vitamina B1.

- También se atribuyen a Algunos medicamentos entre ellos: el tacrolimus y ciclosporina, los daños colaterales como el síndrome de la encefalopatía posterior reversible.

\section{Tipos de Encefalopatías}

Existen varios tipos de encefalopatías, entre las cuales destacan:

- Encefalopatía hepática.

- Encefalopatía urémica.

- Encefalopatía de Hashimoto.

- Encefalopatía de Lyme.

- Encefalopatía de Wernicke.

- Encefalopatía mitocondrial.

- Encefalopatía espongiforme bovina.

\section{Síntomas}

La alteración en el estado mental es la condición común de todos los tipos de encefalopatías, dicha alteraciones pueden manifestarse de forma sutil e ir progresivamente empeorando durante varios años gradualmente lento. Mientras que en otros casos puede manifestarsede forma obvia y desarrollarse rápidamente. Algunas de las cuales puede causar el como y la muerte.

\section{Síntomas que se manifiestan en las ence- falopatías}

- Letargo.

- Demencia.

- Convulsiones.

- Temblores.

- Espasmos musculares y mialgia.

- Respiración de Cheyne-Stokes, un patrón de respiración alterada visto en ca-

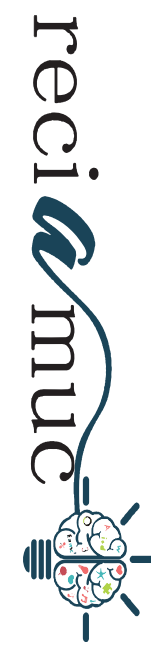


sos de daño cerebral y el coma.

Coma.

\section{Método de diagnóstico}

Las pruebas clínicas que se realizan para el diagnóstico de la encefalopatía se realizan en función de ver el grado de alteración que se presenta y constatación de la enfermedad. El diagnóstico usualmente se lleva a cabo a consecuencia de un estado alterado que se presenta con otra enfermedad principal. En efecto si el paciente presenta diagnostico de enfermedades como anoxia, insuficiencia renal, hepática crónica, entre otras, se efectual cuando hay evidencia de alteraciones del estado mental. En consecuencia, los médicos pueden utilizar varias pruebas diferentes al mismo tiempo para diagnosticar tanto la condición primaria, como la propia encefalopatía. Este enfoque para el diagnóstico es realizado por la mayoría de los médicos, debido a que muchos consideran que la encefalopatía es una complicación que se produce por un problema de salud subyacente.

Pruebas más utilizadas durante los exáme- nes de diagnóstico de las posibles causas primarias:

- La creatinina (para evaluar la función renal).

- Análisis de sangre y otros fluidos corporales.

- Recuento sanguíneo completo.

- La presión arterial.

- Pruebas metabólicas.

- Medicamentos o niveles de toxinas.

- Encefalograma.

- El análisis de autoanticuerpos.

- Ecografía Doppler.

- TC y MRI.

- Revisión de los medicamentos y efectos en el paciente.

\section{Tratamiento}

El tratamiento variará de acuerdo a la encefalopatía que presenta el paciente de acuerdo a la enfermedad principal diagnosticada. Se conoce entre los tratamientos:

Tabla 1. Tratamientos según tipos de encefalopatías

\begin{tabular}{|c|c|c|}
\hline \multicolumn{2}{|r|}{ Tipo de Encefalopatía } & Tratamiento \\
\hline 1 & Anoxia a corto plazo & La oxigenoterapia. \\
\hline 2 & Anoxia a largo plazo & La rehabilitación. \\
\hline 3 & $\begin{array}{l}\text { Toxicidad del alcohol a corto } \\
\text { plazo }\end{array}$ & Líquidos intravenosos o ningún tratamiento. \\
\hline 4 & $\begin{array}{l}\text { El abuso crónico de alcohol - } \\
\text { cirrosis o insuficiencia hepática } \\
\text { crónica. }\end{array}$ & $\begin{array}{l}\text { Lactulosa oral, dieta baja en proteínas, } \\
\text { antibióticos. }\end{array}$ \\
\hline 5 & $\begin{array}{l}\text { Encefalopatía urémica - debido a } \\
\text { la insuficiencia renal. }\end{array}$ & $\begin{array}{l}\text { Corregir la causa fisiológica subyacente, } \\
\text { diálisis, transplante de riñón. }\end{array}$ \\
\hline 6 & Encefalopatía diabética & $\begin{array}{l}\text { La administración de glucosa o la eliminación } \\
\text { de la glucosa en sangre para tratar la } \\
\text { hipoglucemia. }\end{array}$ \\
\hline 7 & Encefalopatía hipo-o hipertensiva & $\begin{array}{l}\text { Medicamentos para aumentar o reducir la } \\
\text { presión arterial. }\end{array}$ \\
\hline
\end{tabular}

Fuente: Autores 2020 
El tratamiento debe orientarse de acuerdo al diagnostico de cada paciente, y la causa que origina e tipo de encefalopatía. En este caso de análisis, se discernirá sobre la encefalopatia uremica, que como se ha dilucidado, son originadas por la insuficiencia renal. Es menester profundizar en los síndromes urémicos, para finalmente con el discernimiento teórico de las encefalopatías generales y de los síndromes urémicos en si mismos, permitan profundizar la comprensión de este estudio, y abrir el campo de análisis de las investigaciones futuras que prevengan la encefalopatia uremica desde su causa raíz.

\section{Síndrome Urémico}

Según (Rondón Nucete, 1998) el término "uremia" se atribuye a Piorry quien en 1840 lo definiera como "orina dentro dela sangre" describiendo las consecuencias de la acumulación decompuestos normalmente eliminados por el riñón; la uremia es elconjunto de síntomas y signos que reflejan la deficienciade todos los sistemas orgánicos, entre ellos laincapacidad renal para conservar la composición y elvolumen del medio interno.

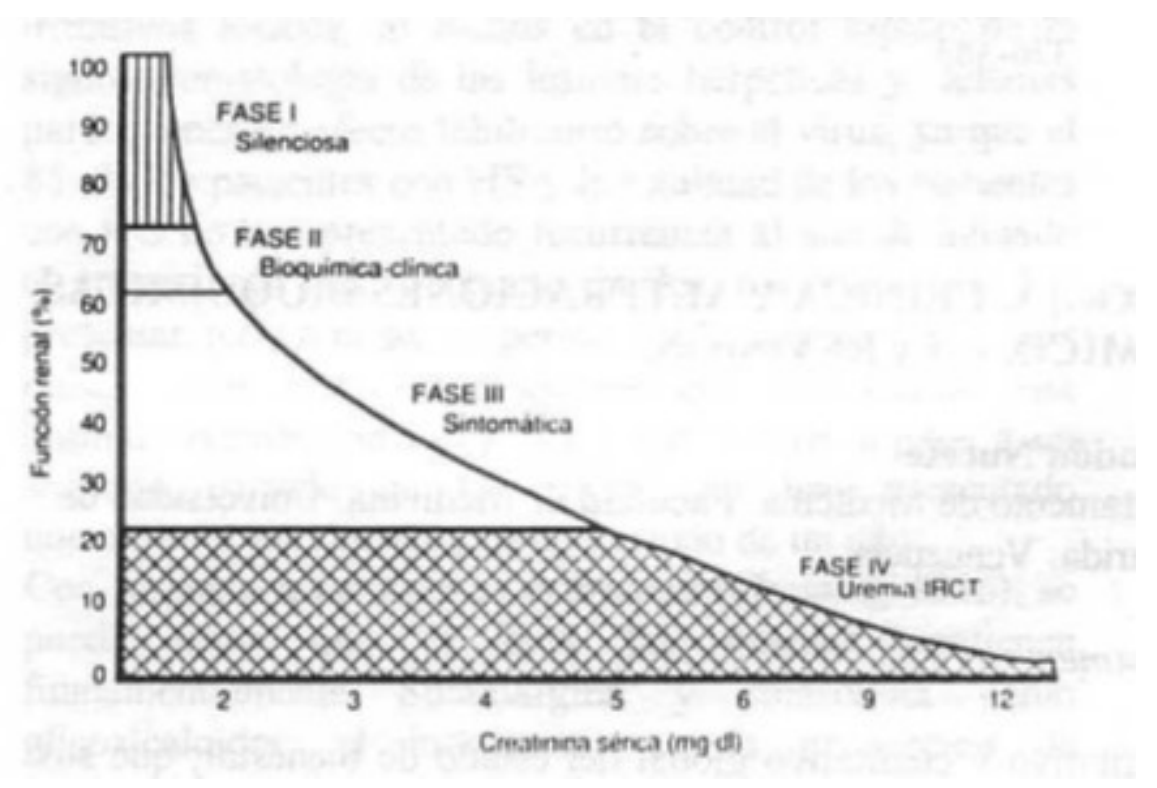

Figura 1. Función Renal

Fuente: (Rondón Nucete, 1998)

En la figura 1 se puede observar que la uremia se corresponde con la fase final en la evolución de la insuficiencia renal crónica, es decir con la insuficiencia renal crónica terminal, solo

Susceptible de tratamiento mediante diálisis y/trasplante renal

En la fase I los riñones pueden perder hasta el $30 \%$ de su masa sin que existan datos clínicos ni bioquímicos, aun cuando se considera que existe una disminución de la reserva renal funcional. En la fase II la función renal se pierde hasta en un $40 \%$, y las cifras de nitrógeno ureico y creatinina séricos se elevan en forma paulatina. La fase III esta dominada por las manifestaciones clínicas y bioquímicas de la insuficiencia renal progresiva tales como una anemia progresiva, hipertensión arterial, edemas, retención azoada, hipertrofia cardiaca radiológica y electrocardiográfica, y aparición gradual de la osteodistrofia renal. La fase IV se corresponde a la insuficiencia renal crónica terminal y es el estadio final de la evolución de la enfermedad renal progresiva, es la fase de uremia clásica la cual aparece cuando la función renal es menor del $5 \%$ de lo normal . Borrero 1989; Ellis et al. 1992citados por (Rondón Nucete, 1998) 
Los signos neurológicos pueden ser episódicos (Ropper, Samuels, \& Klein, 2017) y consisten típicamente en alteraciones del comportamiento, que varían desde la obnubilación (con o sin signos de demencia) al coma, y las convulsiones. Se han comunicado otros signos clínicos tales como tremores musculares, mioclonías, movimientos de cabeza, y debilidad neuromuscular difusa (Bagley, 2005).

Las lesiones renales reportadas en asociación a la encefalopatía incluyen nefroesclerosis, nefrocalcinosis, fibrosisperitubular, degeneración tubular,pielonefritis, infarto renal e hidronefrosis (Wolf, 1980)

\section{Encefalopatía Urémica}

El cuadro clínico según (Osso, 2000) es inespecífico y, como todas las encefalopatías metabólicas, y especialmente cuando se desarrollan rápidamente, pueden producir:

- Acidosis e hiperpnea asociada.

- Delirio florido.

- Confusión progresiva, signos neurológicos focales y convulsiones.

- Cambios motores hasta hemiparesias.

- La tetania es frecuente.
- Asterixis intenso con mioclonias multifocales.

- Las funciones pupilares rara vez se hallan alterados.

La Encefalopatia uremica se puede instalar lentamente y pasar desapercibida de allí la importancia de detectar los síntomas y su evolución:

- Anorexia Vómitos Prurito.

- Nauseas Lentitud Desorientación.

- Insomnio Fatiga fácil Confusión.

- Inquietud, intranquilidad Somnolencia.

- Disminución de la capacidad de atención Inversión del ritmo del sueño Palabra entrecortada.

- Dificultad para manejar ideas.

- Paranoia Comportamiento grotesco.

- Disminución del interés sexual Disminución de las funciones cognitivas Mioclonias.

- Incapacidad para desarrollar ideas abstractas Aseriáis.

- Convulsiones.

- Estupor y coma.

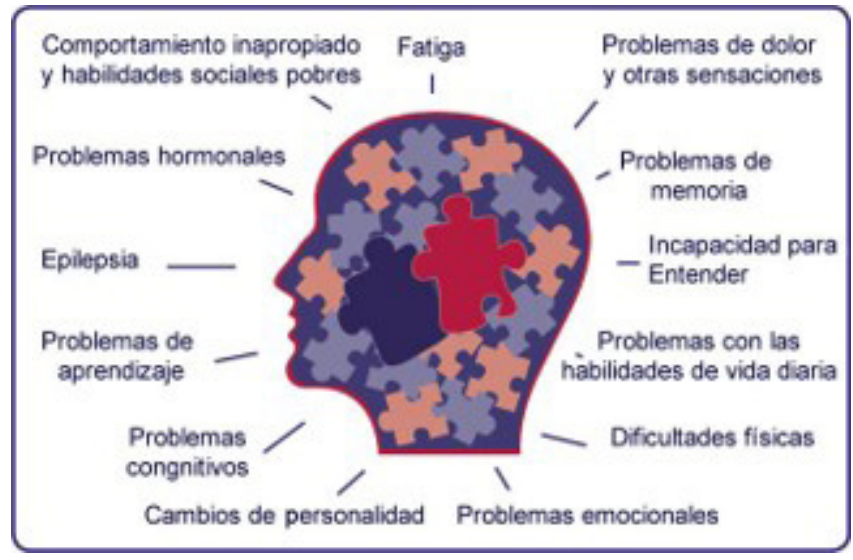

Figura 2. Síntomas de la Encefalopatía

Fuente: Autores 2020 
La pronta identificación de uremia como la causa de la encefalopatía es esencial porque los síntomas son fácilmente reversibles tras el inicio de la diálisis. A medida que la encefalopatía urémica avanza, los pacientes pueden desarrollar micologías, asterixis, convulsiones, estupor y estado coma.
La encefalopatía urémica puede ocurrir en un paciente afectado con una lesión renal aguda o insuficiencia renal crónica de cualquier etiología.

Tabla 2. Evolución de los síntomas de la Encefalopatía Urémica

\begin{tabular}{|l|l|l|}
\hline \multicolumn{2}{|l}{ Síntomas leves } & Síntomas más severos: \\
\hline $\mathbf{1}$ & Anorexia & Comportamiento extraño \\
\hline $\mathbf{2}$ & Náusea & Vómito \\
\hline $\mathbf{3}$ & Inquietud & Inestabilidad emocional \\
\hline $\mathbf{4}$ & Somnolencia & Desorientación \\
\hline $\mathbf{5}$ & $\begin{array}{l}\text { Disminución de la capacidad para } \\
\text { concentrarse }\end{array}$ & Confusión \\
\hline $\mathbf{6}$ & Funciones cognitivas lentas & Disminución de la función cognitiva \\
\hline
\end{tabular}

Fuente: Autores 2020

Tratamiento de la encefalopatía urémica

La presencia de una encefalopatía urémica en un paciente, ya sea con insuficiencia renal aguda o insuficiencia renal crónica, es una señal de alarma para el inicio de la terapia de diálisis: hemodiálisis, diálisis peritoneal o la terapia de reemplazo renal continúa. En los pacientes con enfermedad renal en etapa terminal, las alteraciones del EEG generalmente mejoran después de varios meses, pero pueden no normalizarse por completo.

En el cuidado estándar de cualquier paciente con enfermedad renal terminal también se incluyen los siguientes factores de tratamiento:
- Adecuación de la diálisis.

- Corrección de la anemia.

- Regulación del metabolismo del calcio y el fosfato.

\section{Complicaciones}

Las complicaciones de la encefalopatía pueden variar de ninguna a profundas alteraciones mentales que conducen a la muerte. Además, muchos investigadores consideran que la encefalopatía por sí sola es una complicación que surge de un problema de salud primario. A continuación se listan algunos tipos de encefalopatía y sus principales complicaciones asociadas: 
Tabla 3. Complicaciones Asociadas según tipo de encefalopatía

\begin{tabular}{|c|c|c|}
\hline & Tipo de Encefalopatia & Complicaciones Asociadas \\
\hline 1 & Encefalopatía hepática & Edema cerebral con herniación, coma, muerte. \\
\hline 2 & Encefalopatía metabólica & $\begin{array}{l}\text { Irritabilidad, letargo, depresión, temblores, en } \\
\text { ocasiones el coma o la muerte. }\end{array}$ \\
\hline 3 & Encefalopatía urémica & $\begin{array}{l}\text { Letargo, alucinaciones, estupor, espasmos } \\
\text { musculares, convulsiones, muerte. }\end{array}$ \\
\hline 4 & La encefalopatía de Hashimoto & Confusión, intolerancia al calor, demencia. \\
\hline 5 & La encefalopatía de Wernicke & $\begin{array}{l}\text { Confusión mental, pérdida de memoria, disminución } \\
\text { de la capacidad para mover los ojos. }\end{array}$ \\
\hline 6 & Encefalopatía límbica & $\begin{array}{l}\text { Pérdidas de memoria, sobre todo en la memoria a } \\
\text { largo plazo, comportamiento variable, emocional y } \\
\text { agitado, convulsiones, dificultad para controlar las } \\
\text { extremidades, fatiga y problemas de visión. }\end{array}$ \\
\hline 7 & Encefalopatía glicina & $\begin{array}{l}\text { Progresiva falta de energía, dificultades en la } \\
\text { alimentación, un tono muscular débil, movimientos } \\
\text { espasmódicos anormales y problemas con la } \\
\text { respiración potencialmente mortales. }\end{array}$ \\
\hline 8 & Encefalopatía hipertensiva & $\begin{array}{l}\text { Hipertensión severa, inflamación aguda del riñón o } \\
\text { nefritis, y la disfunción cerebral }\end{array}$ \\
\hline 9 & Encefalopatía isquémica hipoxia & $\begin{array}{l}\text { Pérdida de la memoria, descontrol de los movimientos } \\
\text { y aumentos en la frecuencia cardíaca }\end{array}$ \\
\hline 10 & Encefalopatía estática & $\begin{array}{l}\text { Parálisis cerebral, problemas de aprendizaje, retraso } \\
\text { mental, autismo, retrasos del habla, déficit de } \\
\text { atención, problemas de audición y la visión }\end{array}$ \\
\hline 11 & $\begin{array}{l}\text { Encefalopatía espongiforme } \\
\text { transmisible }\end{array}$ & $\begin{array}{l}\text { Deterioro de la función cerebral, incluyendo cambios } \\
\text { en la memoria, cambios de personalidad y problemas } \\
\text { de movimiento que empeoran con el tiempo }\end{array}$ \\
\hline 12 & Encefalopatía neonatal & $\begin{array}{l}\text { Reflejos frenados, tono muscular deficiente, mala } \\
\text { alimentación, convulsiones y dificultad para respirar. }\end{array}$ \\
\hline
\end{tabular}

Fuente: Autores 2020

\section{Pronóstico}

Las encefalopatías metabólicas son habitualmente reversibles, aunque la encefalopatía hipóxico-isquémica es una importante excepción. Es fundamental el cuidado de soporte que incluye el balance hidroelectrolítico, la nutrición y la suplementación de vitaminas. La agitación puede requerir sedación. Las benzodiacepinas de acción corta (midazolam, loracepam) y el haloperidol son las drogas de elección. El tratamiento del dolor puede reducir la agitación.

El pronóstico para un paciente con encefalopatía depende de las causas iniciales y, en general, la cantidad de tiempo que se necesita para invertir, detener o inhibir las causas. En consecuencia, el pronóstico varía de un paciente a otro y oscila entre la recuperación completa a un mal pronóstico, que a menudo conduce a un daño cerebral permanente o la muerte. Este pronóstico muy variable está ejemplificado por los 
pacientes que reciben la encefalopatía de hipoglucemia. Si los pacientes con hipoglucemia son tratados ante los primeros signos de encefalopatía, por ejemplo, irritabilidad y confusión leve, la mayoría de ellos se recupera completamente. Los retrasos de horas o días en la corrección de la hipoglucemia pueden provocar convulsiones o coma, así como una recuperación parcial. Un largo retraso o múltiples retrasos en el tratamiento pueden llevar a un mal pronóstico, con graves daños cerebrales, el coma o la muerte.

Aunque los síntomas y el marco de tiempo varían ampliamente de paciente a paciente y de acuerdo con las causas iniciales de la encefalopatía, el pronóstico de cada caso por lo general sigue el patrón descrito anteriormente en el ejemplo hipoglucémico y depende de la extensión y la rapidez con la que se trata la causa subyacente.

Causas de encefalopatías urémicas en los pacientes en terapia intensiva:

- Anormalidades metabólicas.

- Sepsis.

- Encefalopatía hepática.

- Insuficiencia renal y síndrome pos dialítico.

- Anormalidades electrolíticas: sodio, calcio, magnesio, fosfato, equilibrio ácido base.

- Encefalopatía Hipóxico-Isquémica.

- Hipo e hiperglicemia.

- Encefalopatía hipertensiva.

- Deficiencia de vitaminas.

- Trastornos endocrinos: hipertiroidismo, hipotiroidismo, insuficiencia suprarrenal.

- Drogas (incluyendo supresión) Alcohol, sedantes, analgésicos, psicotrópicos.

- Infecciones del Sistema Nervioso Central:
- Meningitis.

- Encefalitis.

- Abscesos cerebrales.

- Tromboflebitis séptica.

- Lesiones estructurales cerebrales

- Accidente cerebrovascular (ACV).

- Vasculitis primaria o sistémica.

- Hematoma subdural.

- Trauma encefalocraneano.

- Tumores cerebrales.

- Estados convulsivos.

- Encefalopatía aguda de terapia intensiva.

\section{Epidemiología}

Las complicaciones neurológicas son frecuentes en UTI, pero habitualmente pasan desapercibidas debido a que los pacientes están intubados, sedados o recibiendo drogas bloqueantes neuromusculares. "En 1989, Isensee y col. presentaron un informe sobre 100 pacientes consecutivos admitidos a una UTI médica. El 33\% de los pacientes ingresados por problemas no neurológicos desarrollaron un desorden neurológico agudo.

Los pacientes con mayor riesgo de desarrollar una encefalopatía metabólica son aquéllos con fallas orgánicas múltiples, los ancianos por encima de los 60 años, aquéllos que reciben drogas con toxicidad potencial sobre el sistema nervioso central, y aquéllos con severas deficiencias nutricionales tales como los pacientes neoplásicos o alcohólicos." (Lovesio, 2006)

Los pacientes con encefalopatías tienen una mortalidad mayor, y una estadía más prolongada en Cuidados intensivos que aquellos paciente que no la presentan; la misma pueden ser la primera manifestación de una enfermedad sistémica, como hemos

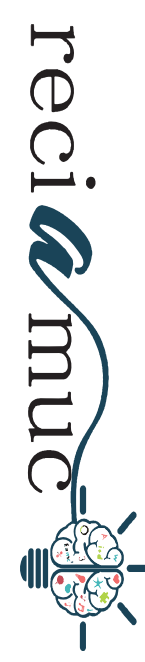


mencionado, su reconocimiento precoz y pronto tratamiento puede mejorar el pronóstico.

\section{Conclusiones}

A modo de conclusión podemos deducir que los estudios, investigaciones, y aportes teóricos sobre las encefalopatías, son necesarios para generar mayor conocimiento en torno a sus casusas y por lo tanto permitir un diagnostico temprano de la enfermedad, ya que como se ha visto en el estudio, la misma puede prevenirse con un diagnostico propico y oportuno; la pronta identificación de uremia como la causa de la encefalopatía es esencial porque los síntomas son fácilmente reversibles tras el inicio de la diálisis, aunado a revertir daños drásticos como el coma o hasta la muerte.

De igual forma, son requeridos los aportes teóricos en función de generar en los especialistas el vasto conocimiento que permita solventar el problema que se presenta en el diagnostico diferencial, cuando el paciente ha recibido drogas que alteran las funciones del Sistema Nervioso Central o cuando el paciente además de insuficiencia renal presenta insuficiencia hepática. Muchos de estas causas pueden prevenirse, detener o limitar la probabilidad de desarrollar cualquiera de las causas que la provocan. Cuando una persona desarrolla una encefalopatía, mientras más rápido se trate la causa subyacente, más probable será evitar una encefalopatía severa.

En el caso de la encefalopatía urémica: debe tratarse con diálisis programada tomar todos los medicamentos según las indicaciones mencionadas y debe evaluarse frecuentemente el estado mental y su evolución.

Los métodos para la prevención de la encefalopatía son casi tan numerosos como las causas subyacentes, sin embargo, casi todos los casos pueden prevenirse, más si se trata de la encefalopatia uremica. Los estudios e investigaciones en esta materia se deben proseguir en cuanto al desarrollo de métodos preventivos que permitan detener a tiempo la evolución de esta complicación del estado mental, a causa de infecciones renales, para ser tratada previamente, conjunto a la enfermedad principal, y sobretodo para orientar a la población en general de los diversos factores que la hacen propensa a sufrir tal enfermedad.

\section{Bibliografía}

Bagley, R. S. (2005). Clinical features of important and common diseases involving the intracranial nervous system of dogs and cats: Metabolic encephalopathies. Fundamentals of Veterinary Clinical Neurology, 129-131.

Cuddon, P. A. (1996, Julio). Metabolic Encephalopathies. The veterinary clinics of Nort America. Small animal practice, 26(4), 893-923.

Cusicanqui, M. I., \& Fernandez C, J. C. (2011). Encefalopatía Urémica. Revista de Actualización Clínica, 11, 565-568.

Lovesio, C. (2006). Medicina Intensiva. Rosario - Argentina: Corpus.

Navas, C. A., \& González, J. (2004). Complicaciones Neurológicas en Cuidado Intensivo. Asociación Colombiana de Neurología, 145-166.

Osso, J. L. (2000). Asociación Regional de Diálisis y Transplantes Renales de Capital Federal y Provincia de Buenos Aires. Retrieved Enero 2020, from https://www.renal.org.ar/recursos/recursos_nef_ clinica_3_insuficiencia_cronica_1_12.php

Pellegrino, F. C. (2009). Encefalopatía asociada a trastornos renales en perros y gatos. AN. VET. (MURCIA), 25, 47-57.

Rondón Nucete, M. (1998). Conceptos actuales sobre la fisipatología, clínica y alteraciones bioquimicas del síndrome urémico. MedULA, Revista de Facultad de Medicina, Universidad de Los Andes., 7(1-4), 35-41.

Ropper, A. H., Samuels, M. A., \& Klein, J. P. (2017). Adams y Victor. Principios de Neurología. (10ma ed.). Ciudad de México: McGRAW-HILL/INTERAMERICANA EDITORES, S.A. de C.V.

Wolf, A. M. (1980). Canine Uremic Encephalopathy. Journal American Animal Hospital Association, 16, 735-738. 


\section{CITAR ESTE ARTICULO:}

Sebastian de Mora Flores, J., Benítez Ormaza, Y., Gavilánez Toalombo, E., \& Alvarez Perez, E. (2020). Encefalopatía urémica. Causas y tratamiento. RECIMAUC, 4(1), 148-159. doi:reciamuc/4.(1).enero.2020.148-159

\section{(c) (1) $\$(0)$ BY NC SA}

RECONOCIMIENTO-NOCOMERCIAL-COMPARTIRIGUAL CC BY-NC-SA

ESTA LICENCIA PERMITE A OTROS ENTREMEZCLAR, AJUSTARY $Y$ CONSTRUIR A PARTIR DE SU OBRA CON FINES NO COMERCIALES, SIEMPRE Y CUANDO LE RECONOZCAN LA AUTORÍA Y SUS NUEVAS CREACIONES ESTÉN BAJO UNA LICENCIA CON LOS MISMOS TÉRMINOS. 